\title{
Enumeration of walks reaching a line
}

\author{
Philippe Nadeau
}

Laboratoire de Recherche en informatique, Bât. 490, Université Paris Sud, 91405 ORSAY Cedex, France

\begin{abstract}
We enumerate walks in the plane $\mathbb{R}^{2}$, with steps East and North, that stop as soon as they reach a given line; these walks are counted according to the distance of the line to the origin, and we study the asymptotic behavior when the line has a fixed slope and moves away from the origin. When the line has a rational slope, we study a more general class of walks, and give exact as well as asymptotic enumerative results ; for this, we define a nice bijection from our walks to words of a rational language. For a general slope, asymptotic results are obtained; in this case, the method employed leads us to find asymptotic results for a wider class of walks in $\mathbb{R}^{m}$.
\end{abstract}

Keywords: walk, generating function, rational language, singularity analysis

\section{Introduction}

In this work we consider primarily two classes of walks in the plane $\mathbb{R}^{2}$, noted $\mathcal{W}_{a, \delta}^{+}$and $\mathcal{W}_{a, \delta}^{-}$, defined in the following manner:

Definition 1 Let $a \in\left[0,1\left[\right.\right.$ and $\delta \geqslant 0$ be real numbers. We denote by $\mathcal{D}_{a, \delta}$ the line of $\mathbb{R}^{2}$ with slope $-a$, going through the point $(\delta, 0)$. An equation of $\mathcal{D}_{a, \delta}$ is $y=-a(x-\delta)$. We denote by $\mathcal{W}_{a, \delta}^{+}\left(\operatorname{resp} . \mathcal{W}_{a, \delta}^{-}\right)$ the set of walks in the plane $\mathbb{R}^{2}$ starting at the origin $O=(0,0)$ with steps East or North, which end as soon as they reach the open (resp. closed) half plane above $\mathcal{D}_{a, \delta}$. The cardinalities of the sets $\mathcal{W}_{a, \delta}^{+}$and $\mathcal{W}_{a, \delta}^{-}$are denoted respectively by $W_{a, \delta}^{+}$and $W_{a, \delta}^{-}$.

These definitions are illustrated on Figure 1.

These walks stop as soon as they cross the line $\mathcal{D}_{a, \delta}$, those in $\mathcal{W}_{a, \delta}^{+}$having to go strictly beyond the line, whereas those in $\mathcal{W}_{a, \delta}^{-}$stop on it if they happen to touch it. We are interested in the enumeration of these walks according to the parameter $\delta$; that is, we fix the slope $-a$ of the line $\mathcal{D}_{a, \delta}$, and study the numbers $W_{a, \delta}^{+}$and $W_{a, \delta}^{-}$in function of $\delta$. Note that, up to a constant factor $a, \delta$ represents the distance of the line $\mathcal{D}_{a, \delta}$ to the origin.

We can now state our first theorem which gives all asymptotic results for $W_{a, \delta}^{+}$and $W_{a, \delta}^{-}$when $\delta$ goes to infinity.

Theorem 1 Let $a \in] 0,1]$, and let $\lambda$ be the unique positive solution to the equation $\lambda^{-1}+\lambda^{-1 / a}=1$.

If $a=p / q>0$ is a fixed rational number, where $p$ and $q$ are relatively prime positive integers, then the asymptotic approximations

$W_{a, \delta}^{+} \underset{\infty}{\sim} \frac{a}{p\left(1-\lambda^{-1 / p}\right)} \cdot \frac{1}{1-(1-a) \lambda^{-1}} \lambda^{\lfloor p \delta\rfloor / p} \quad$ and $\quad W_{a, \delta}^{-} \sim \frac{a}{\infty\left(\lambda^{1 / p}-1\right)} \cdot \frac{1}{1-(1-a) \lambda^{-1}} \lambda^{\lfloor p \delta\rfloor / p}$ 1365-8050 @ 2005 Discrete Mathematics and Theoretical Computer Science (DMTCS), Nancy, France 


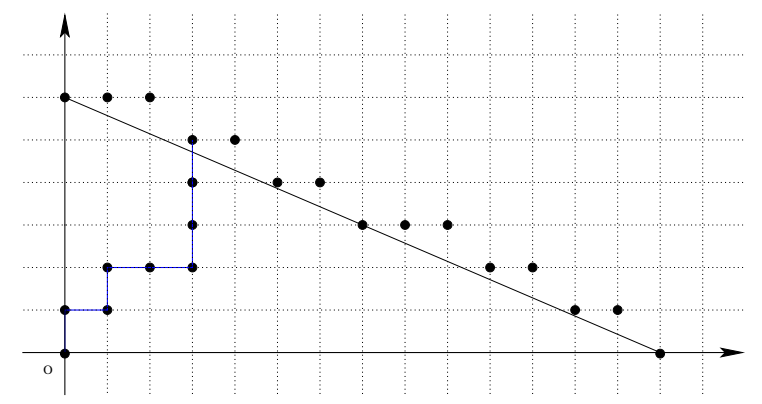

Fig. 1: An example of walk in $\mathcal{W}_{a, \delta}^{+}$with $a=\frac{3}{7}$ and $n=14$.

hold when $\delta$ goes to infinity. If a is irrational, then the asymptotic approximations

$$
W_{a, \delta}^{+} \underset{\infty}{\sim} \frac{a}{\ln \lambda} \cdot \frac{1}{1-(1-a) \lambda^{-1}} \lambda^{\delta} \quad \text { and } \quad W_{a, \delta}^{-} \sim \frac{a}{\infty} \frac{1}{\ln \lambda} \cdot \frac{1}{1-(1-a) \lambda^{-1}} \lambda^{\delta}
$$

hold when $\delta$ goes to infinity.

As this theorem shows, the behavior of $W_{a, \delta}^{+}$and $W_{a, \delta}^{-}$depends on the rationality of the number $a$; if $a$ is rational, then we will find the generating function of the numbers $W_{a, n}^{+}$and $W_{a, n}^{-}$. In this case, we will actually introduce another class of walks that includes $\mathcal{W}_{a, n}^{+}$and $\mathcal{W}_{a, n}^{-}$and find a bijection that sends walks to words of a rational language; various enumerative and asymptotic results derive from there. In the case of a general $a$, we will proceed differently, and start from an easily obtained functional equation to obtain asymptotic results. Our method is close to Erdös et al. $\left(\mathrm{EHO}^{+} 87\right)$, method that is also applicable to a wider class of walks defined in $\mathbb{R}^{n}$.

\section{Walks reaching a set of points}

As announced in the introduction, we now introduce a new class of walks that will include our original walks when the slope of $\mathcal{D}_{a, \delta}$ is rational. The reader is advised to look at Figure 2 while reading the following definition.

Definition $2\left(V_{d, n}\right.$ and $\left.\mathcal{W}_{d, n}\right)$ Let $d=\left(d_{i}\right)_{i \geqslant 1}$ be an infinite sequence of positive integers, and let $e=$ $\left(e_{i}\right)_{i \in \mathbb{N}}$ be the corresponding sequence of partial sums, defined by $e_{0}=0$ and $e_{k}=d_{1}+d_{2}+\cdots+d_{k}$, for $k \geqslant 1$. We associate to $d$ a set of points $V_{d}$ in the plane, with integer coordinates: the set $V_{d} \subset \mathbb{Z} \times \mathbb{N}$ consists in the origin $O$ together with, for every $k \geqslant 1$, the $d_{k}$ points with $y$-coordinate equal to $k$ and $x$-coordinate in $\llbracket-e_{k},-e_{k-1}-1 \rrbracket$.

For any integer $n, V_{d, n}$ is defined as the translated of $V_{d}$ by the vector $(n, 0)$. That is, $V_{d, n}=V_{d}+(n, 0)$. The generalized set of walks $\mathcal{W}_{d, n}$ consists of the walks that start at the origin $O$, make steps East or North, and have their last points, and no other one, in $V_{d, n}$.

These walks are a generalization of our walks $\mathcal{W}_{a, n}^{+}$and $\mathcal{W}_{a, n}^{-}$. Indeed, let $d_{a}^{+}$and $d_{a}^{-}$be the sequences whose $k$ th terms are given respectively by $\left\lceil\frac{k}{a}\right\rceil-\left\lceil\frac{k-1}{a}\right\rceil$ and $\left\lfloor\frac{k}{a}\right\rfloor-\left\lfloor\frac{k-1}{a}\right\rfloor$. Then we have the following proposition : 


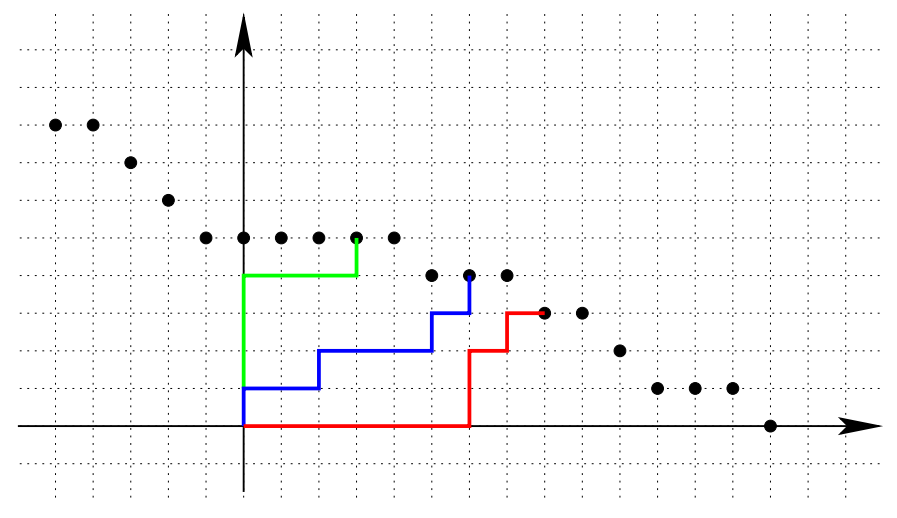

Fig. 2: A set $V_{d, n}$ with examples of walks in $W_{d, n}$. Here $n=14$ and $d=(3,1,2,3,6,1,1, \ldots)$

Proposition 1 For every $n \in \mathbb{N}$ and $a \in] 0,1]$, we have the equalities

$$
\mathcal{W}_{a, n}^{+}=\mathcal{W}_{d_{a}^{+}, n+1} \text { and } \mathcal{W}_{a, n}^{-}=\mathcal{W}_{d_{a}^{-}, n}
$$

An interesting case happens when the sequence $d$ is periodic. It is easy to see that $d_{a}^{+}$and $d_{a}^{-}$are periodic exactly when $a$ is a rational number. If $d=\left(d_{1}, \ldots, d_{p}\right)$ is a finite sequence, we will note $V_{d, n}=V_{\bar{d}, n}$ and $\mathcal{W}_{d, n}=\mathcal{W}_{\bar{d}, n}$, where $\bar{d}$ is the periodic infinite sequence $\left(d_{1}, \ldots, d_{p}, d_{1}, \ldots, d_{p}, \ldots\right)$.

From now on $d$ will stand for a finite sequence $d=\left(d_{1}, \ldots, d_{p}\right)$ of positive integers. We define $q=d_{1}+\cdots d_{p}$, and $a=p / q$. To such a sequence we attach the following language on a finite alphabet (recall that a run in a finite word is a maximal factor composed of identical letters)

Definition 3 (Language $\mathcal{L}_{d}$ ) The language $\mathcal{L}_{d}$ is the set of words $w$ on the alphabet $\Sigma=\left\{a_{0}, a_{1}, \ldots, a_{p-1}\right\}$ that satisfy the following conditions (where we set by convention $d_{0}=d_{p}$ and $a_{p}=a_{0}$ ):

C1. $w$ is the empty word, or its initial letter belongs to $\left\{a_{0}, a_{1}\right\}$

C2. for all $i$, a run of $a_{i}$ in $w$ is terminal or is followed by a run of $a_{i+1}$;

C3. for all $i$, the runs of $a_{i}$ in $w$ are of length at least $d_{i}$; this constraint does not apply to the last run, and, if $w$ begins with $a_{0}$, it does not apply to the first run either.

We can finally state the theorem announced in the introduction:

Theorem 2 Let $n \geqslant 0$ be an integer. There exists an explicit bijection between walks in $\mathcal{W}_{d, n}$ and words of $\mathcal{L}_{d}$ of length $n$.

The language $\mathcal{L}_{d}$ is rational, and we give an unambiguous rational expression that represents it. Then the existence of a bijection as stated in Theorem 2 allows us to explicit the generating function $W_{d}(x)=$ $\sum_{k=0}^{\infty} W_{d, k} x^{k}$ of the sequence $\left(W_{d, n}\right)_{n \in \mathbb{N}}$ :

Theorem 3 The generating function $W_{d}(x)$ has the following expression:

$$
W_{d}(x)=\frac{N(x)}{(1-x)^{p}-x^{q}} \text {, with } N(x)=(1-x)^{p-2}+\sum_{i=1}^{p-2} x^{e_{i}+1}(1-x)^{p-2-i}+\sum_{k=e_{p-1}+1}^{e_{p}-1} x^{k} .
$$


Given a rational function, we can easily have access to asymptotic approximations of the coefficients of its series expansion, and we show that the first part of Theorem 1 can thus be obtained as a consequence of Theorem 3.

In fact, thanks to the bijection of Theorem 2, we can even find the bivariate generating function of the numbers $\left(W_{d, n, k}\right)_{n, k}$ which enumerate walks in $\mathcal{W}_{d, n}$ of length $k$. By the techniques of singularity analysis exposed in chapter 8 of (FS), we can then prove that the average length of a walk in $\mathcal{W}_{d, n}$ is asymptotically $C_{a} \cdot n$ when $n$ goes to infinity, where $C_{a}$ is positive constant depending only on $a$.

\section{Asymptotic results in the general case}

Let $W_{a}^{+}$be the function defined on $\mathbb{R}$ by $W_{a}^{+}(\delta)=1$ if $\delta<0$, and by $W_{a}^{+}(\delta)=W_{a, \delta}^{+}$if $\delta \geqslant 0$. Then, by decomposing walks according to their first step, one shows that $W_{a}^{+}$satisfies the following functional equation:

$$
\forall \delta \geqslant 0, \quad W_{a}^{+}(\delta)=W_{a}^{+}(\delta-1 / a)+W_{a}^{+}(\delta-1) .
$$

This equation and related ones have appeared in various contexts, and have been studied in numerous works, including (CG01; FK74; Pip93). Here we use a method inspired by the paper $\left(\mathrm{EHO}^{+} 87\right)$. This consists in interpreting Equation 1 as a "renewal equation", so that its asymptotic behavior is given by the celebrated Renewal Limit Theorem (RLT) of probability theory; see Feller (Fel71) for all necessary background. Application of the RLT immediately leads to a proof of Theorem 1 as far as $W_{a, \delta}^{+}$is concerned. It is then extended to $W_{a, \delta}^{-}$by finding simple relations between the two numbers.

Our walks have a natural generalization in any dimension. Let $\bar{a}=\left(a_{1}, \ldots, a_{m}\right)$ be a vector in $\mathbb{R}^{m}$, with all coordinates being positive, and $\mathcal{H}_{\delta}$ be the hyperplane of equation $\mathcal{H}_{\delta}: a_{1} x_{1}+\cdots+$ $a_{m-1} x_{m-1}+a_{m}\left(x_{m}-\delta\right)=0$. Then define $W_{\bar{a}, \delta}^{+}\left(\right.$resp. $\left.W_{\bar{a}, \delta}^{-}\right)$to be the numbers of walks in $\mathbb{R}^{m}$ from the origin with steps in $\left\{e_{i}\right\}_{1 \leqslant i \leqslant m}$ defined by the fact that their last points, and no other one, are "above $\mathcal{H}_{\delta}$ " (resp. "above or on $\mathcal{H}_{\delta}$ ").

Assume $1=a_{m} \leqslant a_{1} \leqslant a_{2} \leqslant \ldots \leqslant a_{m-1}$, and let $\lambda$ designate the unique positive solution to $\sum_{i=1}^{m} \lambda^{-a_{i}}=1$. If all $a_{i}$ are rational numbers and we write $a_{i}=p_{i} / q_{i}$ in reduced form for each $i$, we define $q=\operatorname{lcm}\left(q_{i}\right)$. Then the proof of the following theorem is proved along the same lines as described above :

Theorem 4 Let $\lambda$ and $q$ be defined as above. Then we have the following asymptotics when $\delta$ tends to $\infty$ :

(i) if at least one $a_{i}$ is irrational, then

$$
W_{\bar{a}, \delta}^{+} \sim \frac{m-1}{\infty} \frac{m-1}{\ln \lambda \cdot \sum_{i=1}^{m} a_{i} \lambda^{-a_{i}}} \cdot \lambda^{\delta} \quad \text { and } \quad W_{\bar{a}, \delta}^{-} \sim \frac{m-1}{\ln \lambda \cdot \sum_{i=1}^{m} a_{i} \lambda^{-a_{i}}} \cdot \lambda^{\delta},
$$

(ii) and if all $a_{i}$ are rational, then

$$
W_{\bar{a}, \delta}^{+} \underset{\infty}{\sim} \frac{m-1}{q\left(1-\lambda^{-1 / q}\right) \cdot \sum_{i=1}^{m} a_{i} \lambda^{-a_{i}}} \cdot \lambda^{\lfloor q \delta\rfloor / q} \quad \text { and } \quad W_{\bar{a}, \delta}^{-} \underset{\infty}{\sim} \frac{m-1}{q\left(\lambda^{1 / q}-1\right) \cdot \sum_{i=1}^{m} a_{i} \lambda^{-a_{i}}} \cdot \lambda^{\lfloor q \delta\rfloor / q} .
$$

In fact, the same reasoning shows that similar approximations hold in the irrational case when the steps are allowed to be any finite number of non zero vectors with nonnegative coordinates. 


\section{Acknowledgements}

I would like to thank Yves Verhoeven who is at the origin of this work and helped me on many occasions during my researches.

\section{References}

[CG01] V. Choi and M. J. Golin. Lopsided trees. I. Analyses. Algorithmica, 31(3):240-290, 2001. Mathematical analysis of algorithms.

[EHO ${ }^{+}$87] P. Erdős, A. Hildebrand, A. Odlyzko, P. Pudaite, and B. Reznick. The asymptotic behavior of a family of sequences. Pacific J. Math., 126(2):227-241, 1987.

[Fel71] William Feller. An introduction to probability theory and its applications. Vol. II. Second edition. John Wiley \& Sons Inc., New York, 1971.

[FK74] Michael L. Fredman and Donald E. Knuth. Recurrence relations based on minimization. $J$. Math. Anal. Appl., 48:534-559, 1974.

[FS] P. Flajolet and R. Sedgewick. Analytic combinatorics. Book in preparation. Preliminary version available at http://algo.inria.fr/flajolet/Publications/books.html.

[Pip93] Nicholas Pippenger. An elementary approach to some analytic asymptotics. SIAM J. Math. Anal., 24(5):1361-1377, 1993. 
